\title{
A DIGITAL TECHNIQUE TO ESTIMATE POLYNYA CHARACTERISTICS FROM SYNTHETIC APERTURE RADAR SEA-ICE DATA
}

\author{
By JAMES D. LYDEN and ROBERT A. SHUCHMAN
}

(Radar Science Laboratory, Environmental Research Institute of Michigan (ERIM), Ann Arbor,

Michigan 48107, U.S.A.)

\begin{abstract}
A new technique has been developed to estimate digitally the concentration and structure of open-water leads (polynyas) in synthetic aperture radar (SAR) sea-ice data. This procedure consists of smoothing the original SAR sea-ice data to reduce speckle effects, level slicing this smoothed image to produce a binary image consisting of ice and open water, generating the autocorrelation function of this image, and interpreting the autocorrelation function for lead information. Preliminary results indicate that this technique yields useful estimates of lead characteristics, but that additional research is required to evaluate fully its performance.
\end{abstract}

\section{INTRODUCTION}

Synoptic characterization of polynyas or open-water leads in the polar pack ice is important for several reasons. Leads play an important role in the energy balance of the Arctic. The size, orientation, and concentration of leads also determine the navigability of an area. In addition, sequential monitoring of leads provides an estimate of ice dynamics. Synthetic aperture radar (SAR) with its synoptic, all weather, day or night imaging capabilities offers great potential in providing this information. The purpose of this note is to describe a technique which has been developed to extract information on lead characteristics from digital SAR data of sea ice.

A previous study by Bryan and others (1977) examined SAR imagery for lead information. The data used in that study were collected by the Jet Propulsion Laboratory (JPL) L-band $(25 \mathrm{~cm}$ wavelength) SAR system during the 1975 AIDJEX program. The optically processed L-band image films were digitized using a microdensitometer to facilitate digital analysis. The procedure used in the Bryan study consisted of level slicing the digitized data to form binary images representing open water (leads) and ice. The total coricentration of leads was then estimated from histograms of the binary image data. They also estimated lead orientation through examination of the Fourier transforms of the binary imagery. The assumption made in the Bryan study was that there would be a concentration of energy in the Fourier transform with the same orientation as that of the leads. This assumes that the leads are randomly oriented, which is not justified for a large part of the Arctic. The procedure reported in this note does not require this assumption.

\section{APPROACH}

The lead parameters of most interest are open-water concentration, average length and width, orientation, and spatial separation. This information is contained in the autocorrelation of a binary image representing leads and ice only. The procedures followed in generating and analyzing this auto-correlation function are described below.

The data we used in our study were collected in the Beaufort Sea by the STAR-1 SAR system in the fall of 1984. The STAR-1 system is an all-digital SAR which

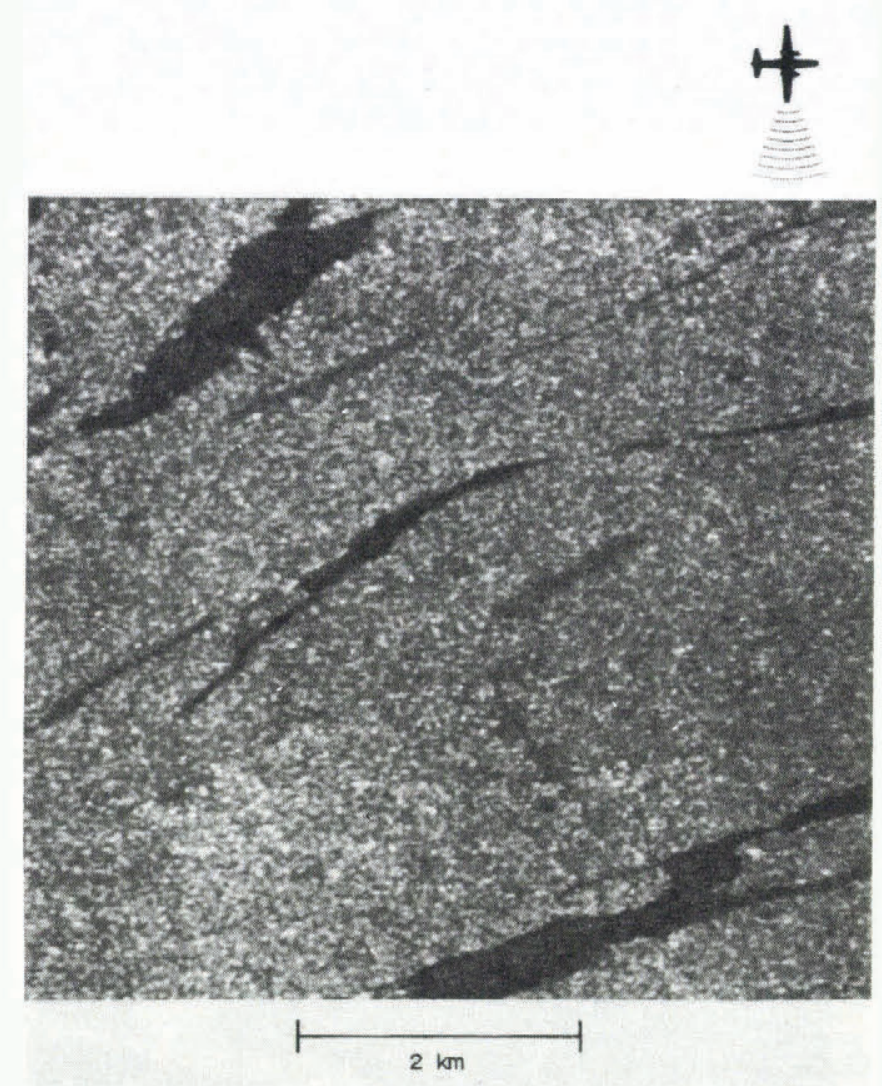

Fig. 1. STAR-1 image used in lead studies; this image was collected in the Beaufort Sea from an altitude of 31000 feet $\left[\begin{array}{ll}10 & 165 \mathrm{~m}\end{array}\right]$ in the fall of 1984 .

operates at X-band $(3.2 \mathrm{~cm}$ wavelength) (Nichols and others, 1986). The STAR-1 system collected data over a $25 \mathrm{~km}$ wide swath at a resolution of $6 \mathrm{~m}$ in both azimuth and slant range. These data were collected with four azimuth looks averaged. A $6 \mathrm{~km} \times 6 \mathrm{~km}$ sub-set image was selected for our analysis and is shown in Figure 1. This image has been re-sampled to $24 \mathrm{~m} \times 24 \mathrm{~m}$ pixels. The open-water leads are imaged as dark or no-return areas. In some of the leads, very young ice has formed which produces a grayish return. For navigation purposes, this very thin ice $(<10 \mathrm{~cm})$ does not present any difficulties so no effort was made at differentiating it from the purely open-water leads. The ice shown in the image is predominantly composed of thick ice floes.

The first step in our procedure was to smooth the data using a $5 \times 5$ pixel window median filter. This smoothed image is presented in Figure 2. Note that most of the speckle-related noise present in the original image has been eliminated. Statistics were generated for both the open-water 


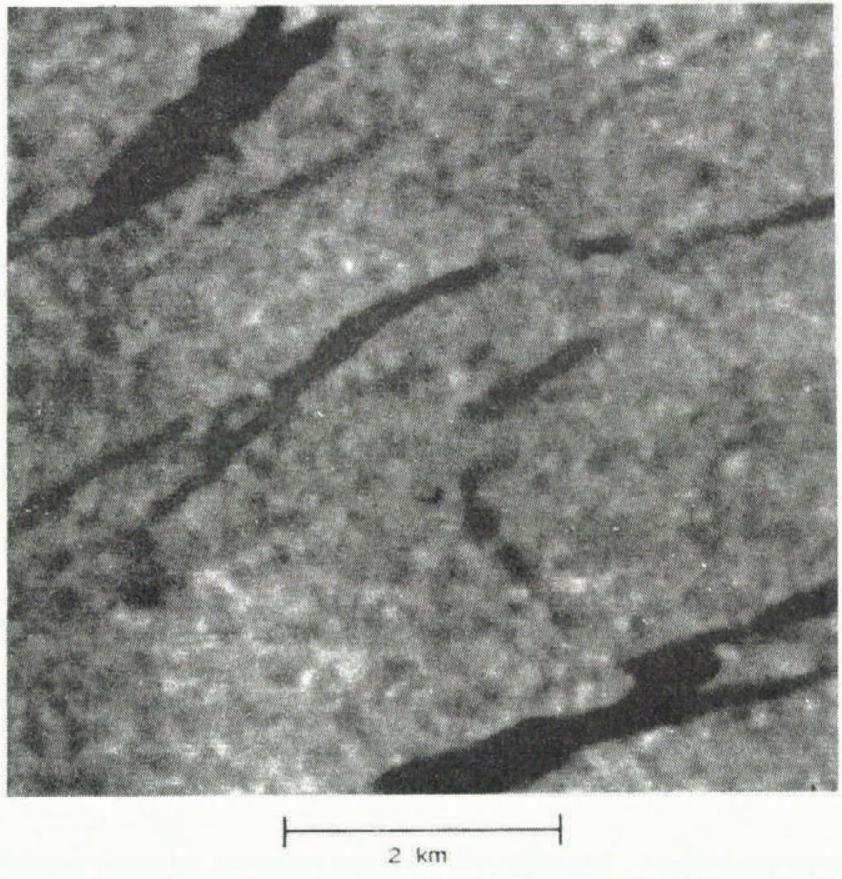

Fig. 2. STAR-1 image after smoothing by a median filter.

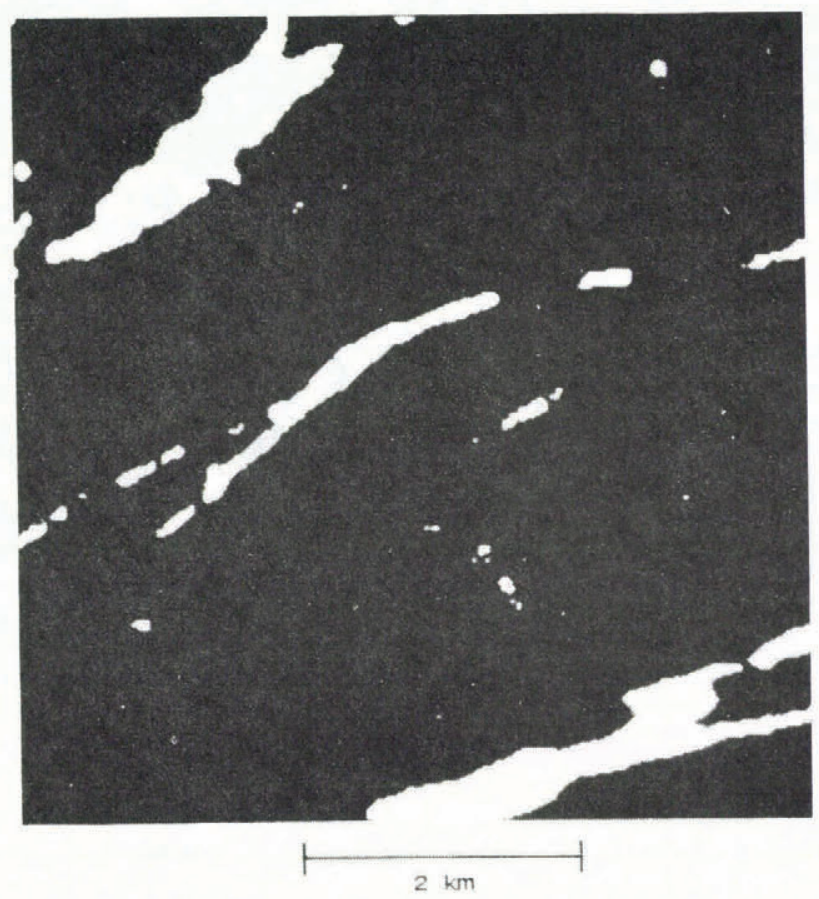

Fig. 3. Binary image showing open-water leads.

and ice areas to determine an appropriate threshold to separate the two. Using this threshold, a binary image was generated with is assigned to the open-water pixels and 0 s to the ice pixels. This binary image is presented in Figure 3. The open-water areas in this image are primarily contained in three leads: two with approximately the same size and one which is about half the width of the others.

The autocorrelation function of this binary image was generated using Fourier transforms and its contour plot is presented in Figure 4. This function was normalized by the number of pixels; therefore, the 0 pixel lag or peak of the autocorrelation function corresponds to the concentration of open water in the image. For this image, a $7.6 \%$ concentration of leads was determined. The average size of the leads can be estimated from the contour plot of the autocorrelation function by noting the position where the fall-off of the autocorrelation tapers. For the function

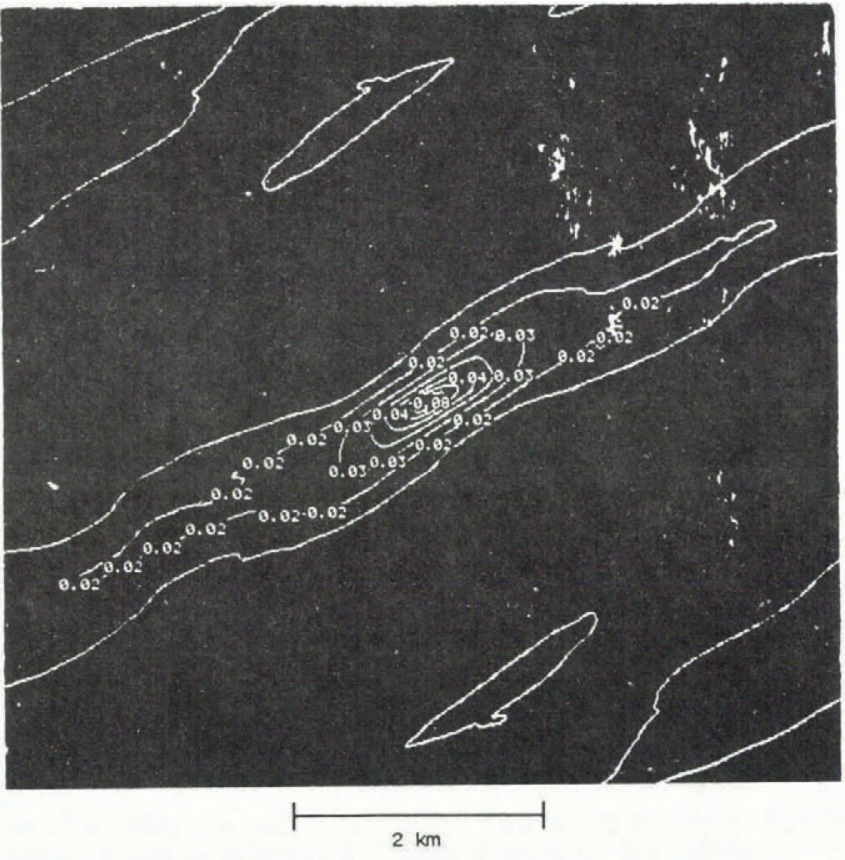

Fig. 4. Contour plot of the autocorrelation function produced from the binary image shown in Figure 3.

plotted in Figure 4, this interpretation gives an average length and width of 120 and 15 pixels or $2.9 \mathrm{~km}$ and $0.4 \mathrm{~km}$, respectively. The total number of open-water pixels can be determined by multiplying the concentration estimate determined above by the number of pixels in the image. Dividing by the average lead size provides an estimate of the number of leads in the image. For this example, the number of leads in the image is calculated to be 2.8 .

The average orientation of the leads is interpreted to be the same as the orientation of the major axis of the autocorrelation function, which for this example is approximately $30^{\circ}$ above horizontal. The last piece of information we are concerned with is the location of the leads relative to one another. Limited information is available from the location of the second-order peaks of the autocorrelation function. Two second-order peaks are visible in Figure 4 and suggest that the leads are primarily separated in a direction perpendicular to their length axis. The second-order peaks indicate separations of 85 and 134 pixels which translates into distances of $2.0 \mathrm{~km}$ and $3.2 \mathrm{~km}$, respectively. These results are consistent with the image in Figure 3.

It should be emphasized that the autocorrelation function contains no information about the phase of the original image and is therefore not unique insofar as the same autocorrelation function may be obtained from a variety of images. This simply means that we cannot reconstruct the original image from the autocorrelation function. It does, however, provide an excellent measure of the lead characteristics of an ice scene. Using the information obtained from Figure 4 , that is, three leads of $15 \times 120$ pixels oriented at $30^{\circ}$ above horizontal with spacings of 85 and 134 pixels, one reconstructed image would look like the one presented in Figure 5. This was produced assuming that one of the three leads was centered in the image. There is a $180^{\circ}$ ambiguity in the locations of the other two leads. Comparison of Figures 5 and 3 indicates that the autocorrelation method provides a good estimate of the open-water leads in the SAR sea-ice image.

\section{CONCLUSIONS}

A new procedure has been developed for digitally estimating the concentration and structure of open-water leads in SAR sea-ice data. This procedure consists of smoothing the original SAR sea-ice data to reduce speckle effects, level slicing this smoothed image to produce a 


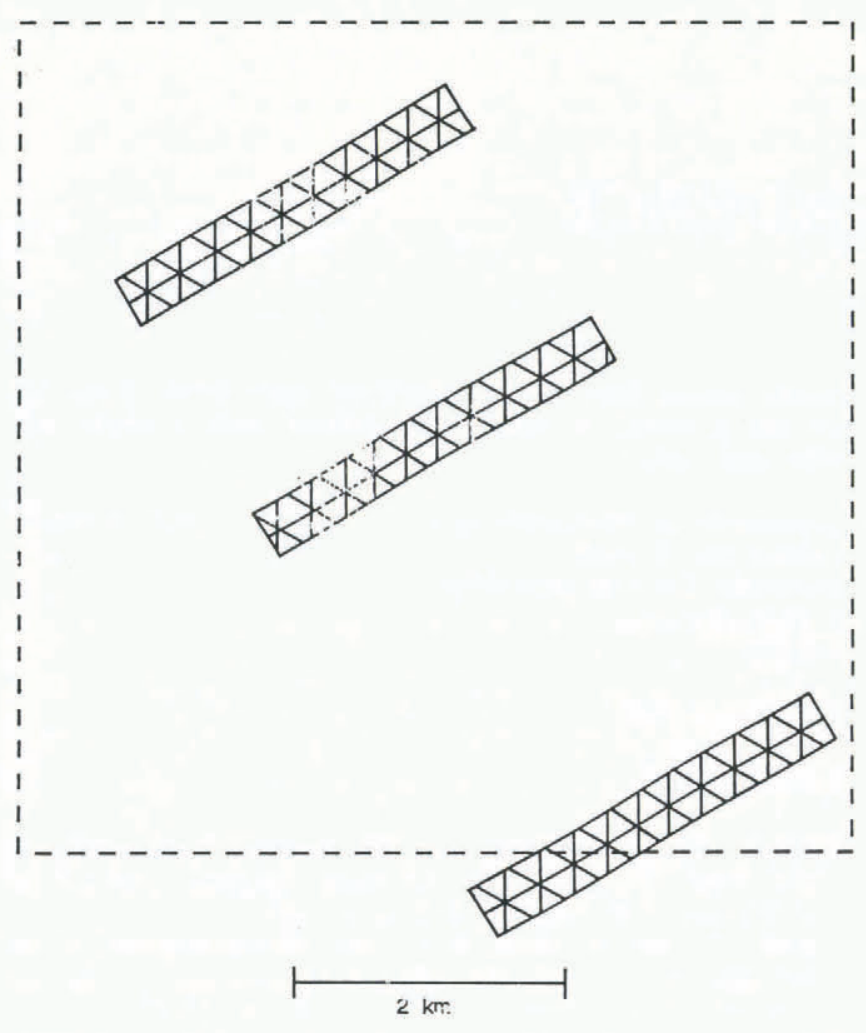

Fig. 5. One possible reconstruction of lead positions from autocorrelation function.

binary image of ice and open water only, generating the autocorrelation of this image, and interpreting the autocorrelation function for lead information.

Results from applying this method to a STAR-1 sea-ice image containing open-water leads are encouraging. Estimates of lead concentration, average size, orientation, and spatial separation were obtained. One realization of the lead locations was generated from these estimates and found to agree well with the original imagery. These results indicate that the lead characteristics of a scene can be adequately characterized by just a few parameters.

Recommendations for additional studies include applying this technique to more complex sea-ice scenes to determine its effectiveness. Intuitively, these would be the conditions where this technique would outperform a human interpreter. In a confused scene, a human interpreter would have problems estimating an average size or orientation. The autocorrelation function, however, provides a statistically based estimate of these parameters.

Additional research also is required to automate fully the above procedure. These studies should examine the selection of the threshold to differentiate ice from open-water or thin-ice regions. In addition, techniques to interpret automatically the autocorrelation function should be investigated.

\section{ACKNOWLEDGEMENT}

This work was supported by the Office of Naval Research (ONR) under contract No. N00014-81-C-0295. The technical monitor for this contract is $\mathrm{Mr} \mathrm{C}$. Luther.

\section{REFERENCES}

Bryan, M.L., and others. 1977. Computer processing of SAR L-band imagery, by M.L. Bryan, W.D. Stromberg, and T.G. Farr. Photogrammetric Engineering and Remote Sensing, Vol. 43, p. 1283-94.

Nichols, A.D., and others. 1986. A SAR for real-time ice reconnaissance, by A.D. Nichols, J.W. Wilhelm, T.W. Gaffield, D.R. Inkster, and S.K. Leung. IEEE Transactions on Geoscience and Remote Sensing, GE-24, p. 383-89. 